$17^{\text {th }}$ International Congress of Metrology, 12002 (2015)

DOI: $10.1051 /$ metrology / 201512002

(C) Owned by the authors, published by EDP Sciences, 2015

\title{
The calibration of static and dynamic performances of PMUs
}

\author{
J.P. Braun ${ }^{1}$, S. Siegenthaler \\ Federal Institute of Metrology METAS, 50 Lindenweg, 3003 Bern-Wabern, Switzerland
}

\begin{abstract}
Résumé. Depuis quelques années on assiste à une accélération du déploiement de PMU dans les réseaux électriques de transmission. Les PMU, comme la plupart des instruments, exigent une infrastructure spécifique qui permet la certification et la vérification de leurs performances métrologiques. Celles-ci sont déterminées par un ensemble de tests statiques et dynamiques décrits dans la norme IEEE C37.118.1. Dans le cadre d'un projet EMRP (European Metrology Research Programme), METAS a développé un système d'étalonnage permettant cette caractérisation. Les performances de ces synchrophaseurs et de leurs systèmes de vérification devront toutefois être améliorées afin de répondre aux futures attentes de l'utilisation de PMU dans les réseaux de distribution. En effet les courtes distances des réseaux de distributions et la présence de perturbations exigent une résolution en phase accrue ainsi qu'une bonne robustesse face à ces perturbations. En vue de répondre à ces nouvelles exigences, METAS a entrepris la modification de son système d'étalonnage. Le but de cet article est de présenter la conception et l'utilisation d'un système d'étalonnage de PMU, ainsi que l'effort entrepris pour l'étalonnage des future PMU pour les réseaux de distribution.
\end{abstract}

\section{Introduction}

Due to a lack of common time reference, the measure of the relative phase angle between the two distant buses in a power system could not be directly measured in the past. Early attempts were made in the 80 s by using the Loran navigation system to synchronise measurements, but time accuracy proved to be a limitation. The advent of the Global Positioning System (GPS) resolved this conundrum and enabled the Phasor Measurement System (PMU) technology. PMUs measure the voltage or current phasor with respect to UTC, simultaneously at any geographical location. Over the last 20 years, PMUs gained acceptance in transmission networks for several applications [1].

The acceptance of PMUs was hindered for a long time by issues such as inter compatibility between devices, the lack of standardised tests and availability of independent test laboratories [2,3]. The first two issues were progressively resolved through normalisation [4,5], and the last one led the National Institute for Standards and Technology (NIST) to establish an independent synchrophasor laboratory [6]. In Europe, the need for a metrological infrastructure for PMUs was also recognised by the metrological community and led to an EMRP project aiming to develop a metrological infrastructure supporting smart grid initiatives [7]. Within this framework, METAS developed a PMU calibrator suitable to test the requirements of standard IEEE C37.118.1.

${ }^{1}$ Email of corresponding author: jean-pierre.braun@metas.ch
PMUs are perceived today by some researchers, to be also capable of playing an important role in distribution networks. The ever increasing penetration of distributed generation could lead to instability in these networks and may require active control. The role of PMUs would be to monitor and later control distribution networks. However, the small dimensions of a distribution network as well as higher level of power quality disturbances demand significant improvements in PMU's performances.

The objective of this paper is to present the nature of the metrological characterisation of PMUs and the calibrator developed by METAS as well as the ongoing effort to upgrade this system for advanced PMUs.

\section{Characterisation of PMUs}

PMUs extract the phasor of a cosinusoidal waveform, voltage or current, with respect to UTC. The signal and its phasor are respectively defined as:

$$
\begin{gathered}
x(t)=X_{m} \cos (\omega t+\varphi) \\
\boldsymbol{X}=\left(X_{\mathrm{m}} / \sqrt{2}\right) e^{\mathrm{j} \varphi}
\end{gathered}
$$

Where $\varphi$ is the instantaneous phase angle relative to a cosine function at nominal system frequency and synchronised to UTC. PMUs report also the instantaneous power frequency and the rate of change of the frequency (ROCOF): 


$$
\begin{gathered}
x(t)=X_{\mathrm{m}} \cos (\psi(t)) \\
f(t)=\frac{1}{2 \pi} \frac{d \psi(t)}{d t} \\
\operatorname{ROCOF}(t)=\frac{d f(t)}{d t}
\end{gathered}
$$

These measurements are reported at one of the following rates $(50 \mathrm{~Hz})$, Fs: 10, 25, 50 frames per second.

Three criteria are used to evaluate PMU measurements, namely the total vector error (TVE), the frequency error (FE) and the rate of frequency change error (RFE). The TVE consists in an error phasor between the measured and the true phasors as shown in Figure 1. This criterion combines the magnitude, phase and timing errors. IEEE C37.118.1 limits the TVE to 1 $\%$. The other two criteria, FE and ROCOF are defined as:

$$
\begin{gathered}
\mathrm{FE}=\left|f_{\text {true }}-f_{\text {measured }}\right| \\
\mathrm{RFE}=\left|\left(\frac{d f}{d t}\right)_{\text {true }}-\left(\frac{d f}{d t}\right)_{\text {measured }}\right|
\end{gathered}
$$

These criteria are used for the various compliance tests outlined in IEEE C37.118.1 which are classified either as steady-state (static) or dynamic. For the former, the values of $X_{\mathrm{m}}, \omega$ and $\varphi$ or the other influences remain at a steady value for the duration of test while in the later they are time variant. The standard sets limits for the TVE, FE and RFE for each given test.

\subsection{Steady-state compliance tests}

- Signal frequency range

- Voltage / current magnitude

- Phase angle

- Harmonic distortion

- Out-of-band interference

The first three tests consist simply in a change of one parameter between two limits with a given increment. In the last two tests, harmonics or interharmonics are added to the power signal as influence quantities. Their magnitude is set at $10 \%$ of the nominal voltage or current.

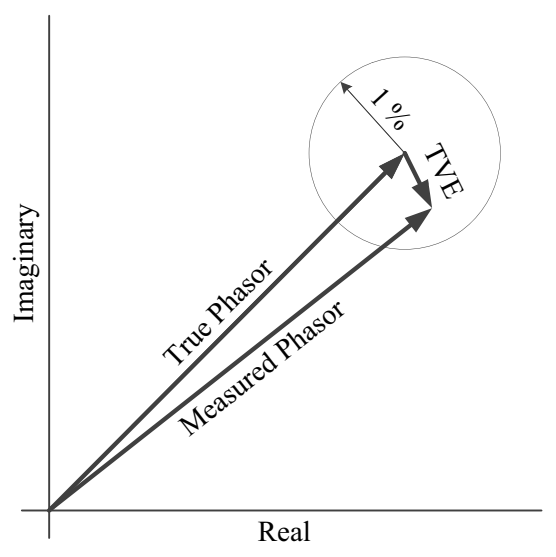

Figure 1. The TVE criterion

\subsection{Dynamic compliance tests}

- Measurement bandwidth (modulation)

- Ramp of system frequency

- Step changes in magnitude or phase

The bandwidth test aims at assessing how well a PMU can track magnitude or phase variations. To do so, the power signal is respectively amplitude or phase modulated at a rate that is linked to the reporting rate. The ramp test assesses a PMU's ability to track a steady increase or decrease in the power frequency. Finally, the step changes permits to establish the time required by a PMU to recover a rapid change of magnitude or phase as well as ringing caused by the step.

\section{The METAS PMU calibrator}

The calibrator was designed as a metrological grade instrument suitable for the full certification or verification of PMUs against and beyond IEEE C37.118.1. It was also conceived to be later used as a calibrator for PMU calibrators that are now appearing in the market. Figure 3 shows the architecture. This one can be divided in four sections: the timing section, the forward and return paths as well the data processing software.

\subsection{The hardware}

The timing section synchronises all waveforms generation and acquisition to UTC. The synchronisation to UTC is achieved either with GPS, IEEE 1588 or IRIGB. The GPS and the IEEE 1588 are the most precise and enable time accuracy in the order of $100 \mathrm{~ns}$. The hardware is realised with NI PXI cards which uses two specific modules for the timing: NI-6653 and 6682. The former is a precision oven temperature controlled 10 $\mathrm{MHz}$ quartz oscillator disciplined to UTC with the later device. This $10 \mathrm{MHz}$ master clock is perfectly aligned to UTC and the time at every sample is thus known. Figure 2 shows the $10 \mathrm{MHz}$ clock as well as the PPS (Pulse Per Second) indicating the passing of the UTC second. The complete timing system was characterised against UTC$\mathrm{CH}$, the Swiss realisation of the UTC. Figure 4 shows the fluctuations of the PPS of the calibrator with respect of UTC-CH when GPS is used as source of time.

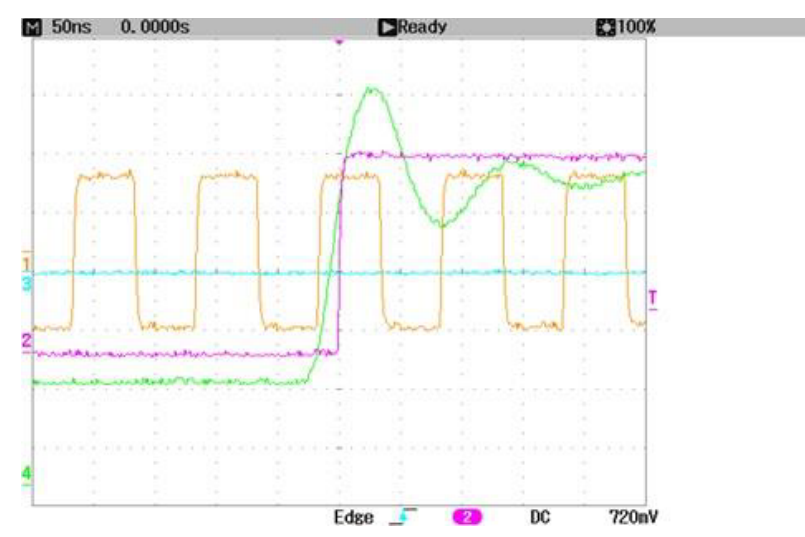

Figure 2. Synchronisation of $10 \mathrm{MHz}$ to UTC with IRIG-B 


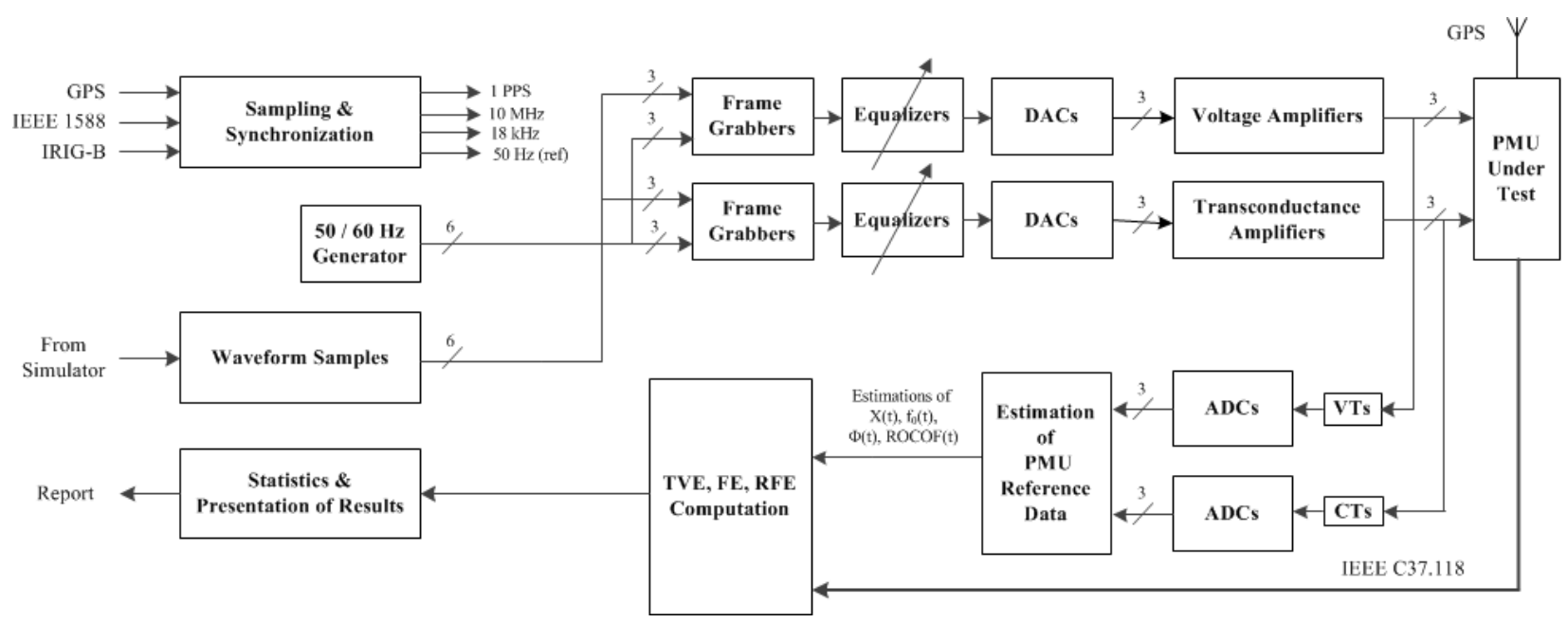

Figure 3. Architecture of the METAS PMU calibrator

The forward path DACs and reverse path ADCs path use a sampling rate of $18 \mathrm{ksps}$ locked to the $10 \mathrm{MHz}$ master clock. The frequency of $18 \mathrm{ksps}$ is a multiple of 50 and $60 \mathrm{~Hz}$ as well as all reporting frame rates for these two power frequencies. The actual three phase (voltage and current) waveform generation is based on frames of $0.1 \mathrm{~s}$ in duration containing either a test signal or a nominal cosinusoidal $50 \mathrm{~Hz}$ signal whose L1 phase L1 is set exactly to zero degree at the passing of the UTC second. The waveforms used for an IEEE C37.118.1 test are also organised in frames of $0.1 \mathrm{~s}$, computed off line, and stored in a hard disk and then streamed during a test. This playback method has the advantage of being fast, but at the expenses of flexibility and several gigabytes of data storage. To avoid any discontinuity with the nominal 50 or $60 \mathrm{~Hz}$ signal, any test signal starts synchronously with the 50 or $60 \mathrm{~Hz}$ standby signal. Additionally, all tests start exactly at the passing of the second in order to be coherent with the PMU's reporting frame. Figure 5 and 6 show two signals used respectively for the harmonic and magnitude step. These test signals are amplified and then applied to the PMU.

The PMU processes the time synchronised signals and reports the phasor (magnitude and phase), frequency and ROCOF at the selected frame rate. All frames are time stamped and can thus be correlated with the signal applied at the PMU's input.

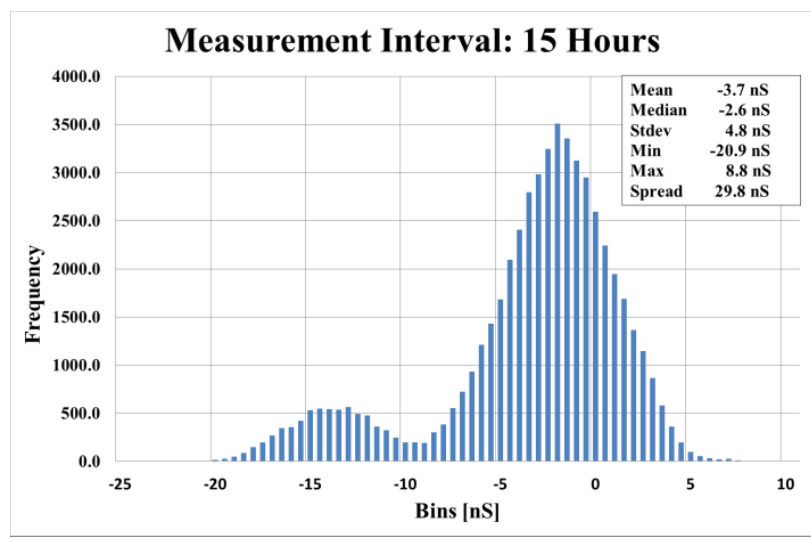

Figure 4. Characterisation of the PPS with respect to UTC-CH
In order to evaluate the PMU data, reference results must be available. The reference data could be created with a reference PMU whose performances exceed those of the DUT. But, as a PMU design is a trade-off between several performances, another approach was chosen. The waveforms present during a test at the input of the PMU are resampled and analysed and then compared with the PMU data. The analysis is specifically tailored for the test at hand. Prior the resampling and analysis the signals are first conditioned with precision shunts and actives voltage dividers. As the resampling uses the same sampling rate of $18 \mathrm{ksps}$ and the same $0.1 \mathrm{~s}$ frame, the exact time corresponding to each sample is known with great precision. It is the accuracy of the return path that determines the overall quality of the calibration.

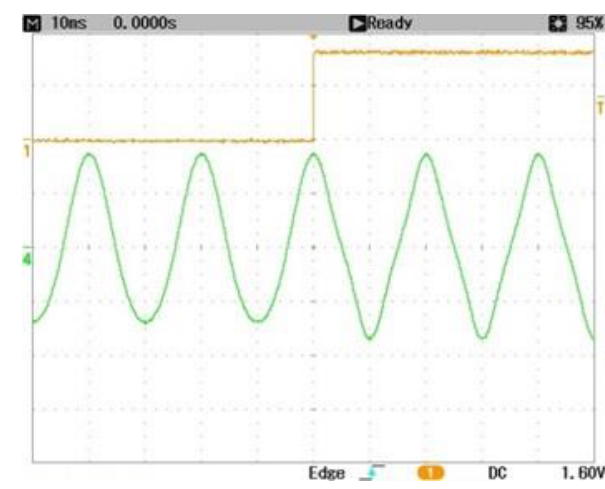

Figure 5. Change from $2^{\text {nd }}$ to $3^{\text {rd }}$ harmonic at passing of second

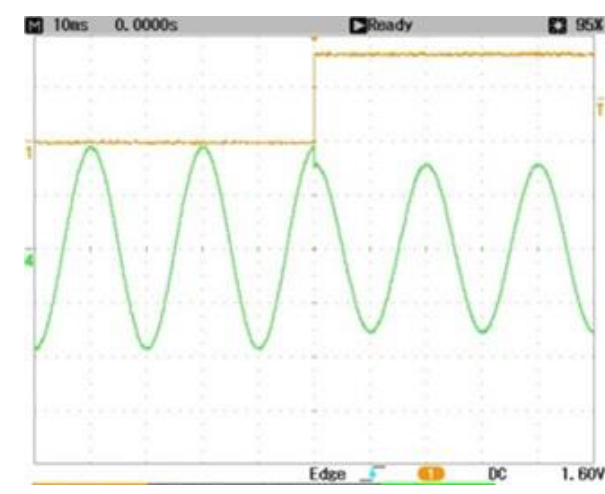

Figure 6. Magnitude step a the passing of second 


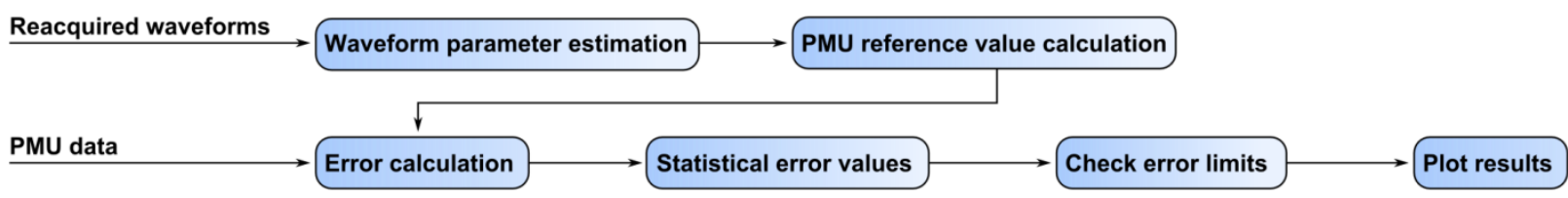

Figure 7. Data processing of sampled waveforms and PMU data

\subsection{Data analysis}

The overall data processing requires several steps in order to assess the PMU data against a test scenario contained in the waveform and to determine its conformity to the IEEE C37.118.1 standard.

\subsubsection{Estimation of waveform parameters}

The waveform parameter estimation uses least square fitting to extract the waveform parameters from the time stamped samples. As all the tests defined in the standard are deterministic, the fitting permits to establish the actual parameters such as magnitude, phase, frequency and ROCOF. The fitting is often facilitated by the fact that some parameters such as modulation frequency do not require extraction as the highly accurate time synchronisation ensures their correctness. For steadystate compliance tests, the fitting uses a linear model with one or two sine waves of known frequency. However, for dynamic tests, the signal may contain modulation forcing the use of iterative methods (nonlinear). However, the convergence is generally fast as the initial conditions are those used in generated waveform (synthetic data). The small differences between the acquired waveform and those generated are caused primarily by the frequency response of the amplifiers.

\subsubsection{PMU reference value calculation}

Once the waveform parameters are established, the PMU reference values are computed using equation 1 to 5. These are the values that the PMUs should report.

\subsubsection{Error calculation}

Is the difference between the PMU measurements and the reference values extracted from the waveform samples: magnitude, phase, frequency and ROCOF.

\subsubsection{Statistical error values}

As the values of TVE, FE and ROCOF are acquired over several seconds, statistical values such as mean, standard deviation, minimum and maximum values are also extracted.

\subsubsection{Check error limits}

The error values between the PMU results and the references values are compared against the limits set by IEEE C37.118.1. A pass/fail evaluation is made.

\section{Examples of some PMU calibrations}

In this section some measurements obtained on a commercial PMU are presented. The PMU was configured with three phases, voltage and current, respectively of $110 \mathrm{~V}$ and $1 \mathrm{~A}$ and a power frequency of $50 \mathrm{~Hz}$. A. The reporting rate of was set to $50 \mathrm{fps}$. But, due to limited space, only the results of phase L1 are presented. Most results include mean, standard deviation and minimum and maximum values of the measurement.

Figure 8 shows the result of a signal frequency range test (steady compliance test). The top graph shows how the TVE is affected by the power frequency. The middle one shows the error on the frequency while the bottom graph shows the error of the ROCOF. The three graph show compliance to IEEE C37.118.1 for this test.

Figure 9 shows the influence on the TVE of a single harmonics whose magnitude is $10 \%$ of the fundamental.
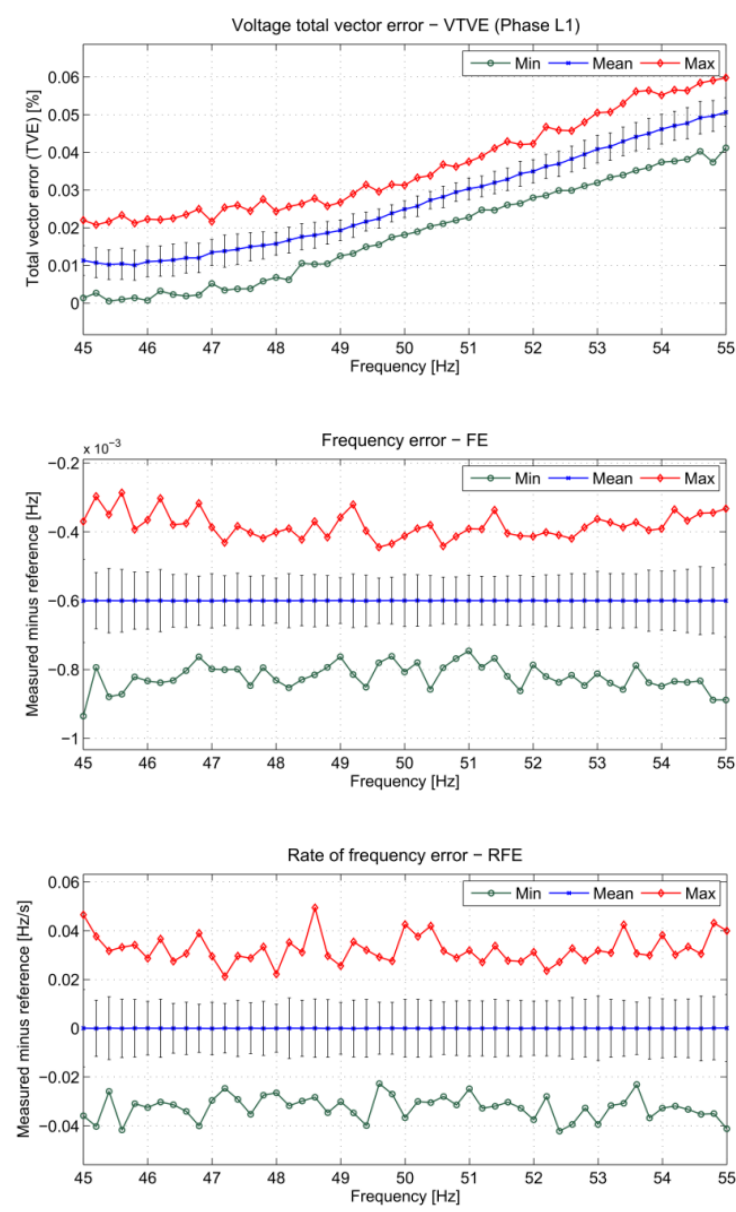

Figure 8. Signal frequency range tests (Steady-state) 


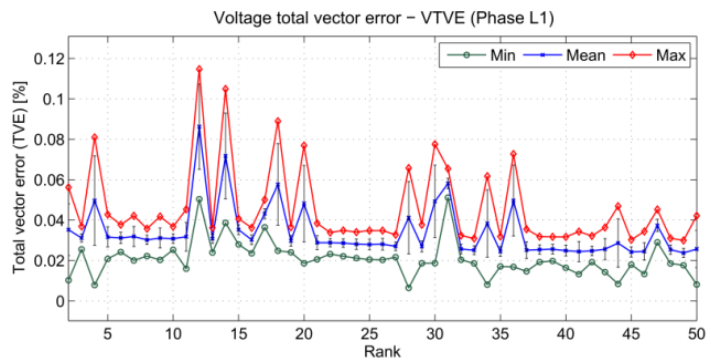

Figure 9. Harmonic test (Steady-state)

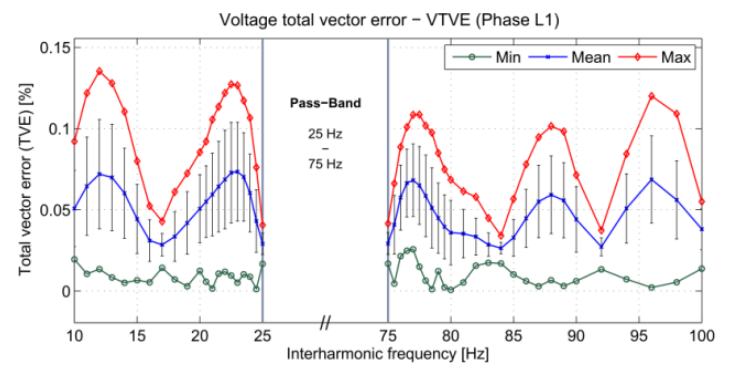

Figure 10. Out-of-band interference (Steady state)

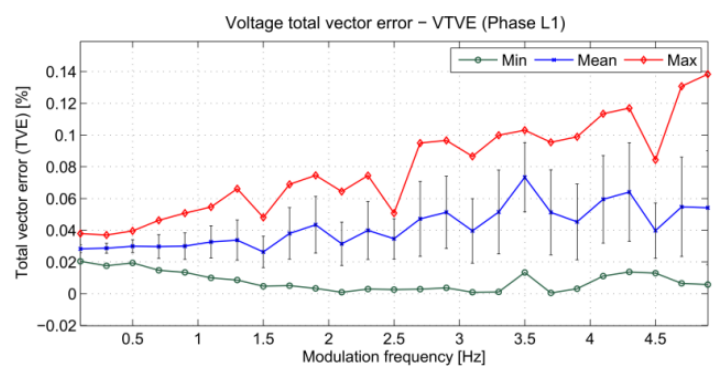

Figure 11. Phase modulation test (dynamic test)

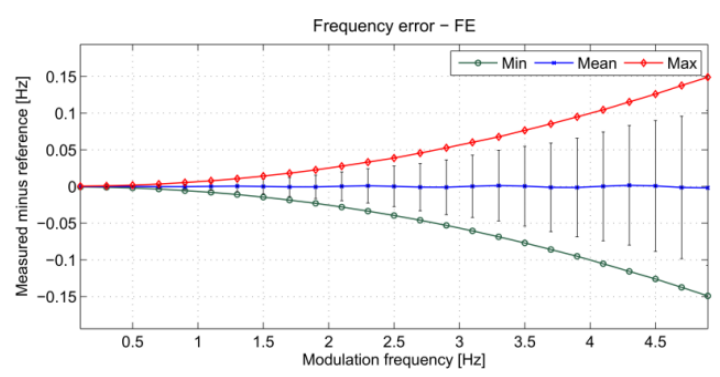

Figure 12. Frequency error during a phase modulation test (dynamic test)

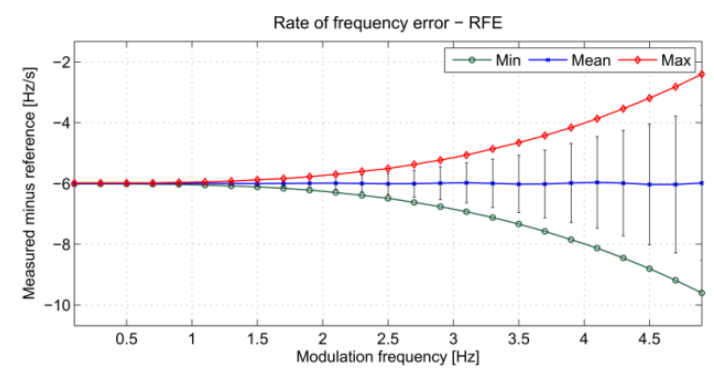

Figure 13. ROCOF error during a phase modulation test (dynamic test)

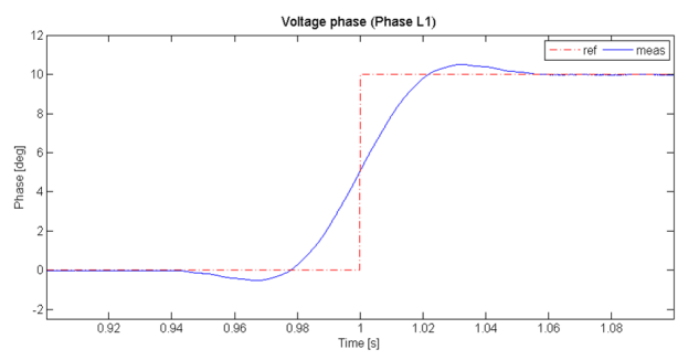

Figure 14. Phase step change test (dynamic test)

Figure 10 shows the influence of intherharmonics outside the $25 \mathrm{~Hz}$ bandwidth of the PMU (Reporting rate of 50 frames per second). This test proved to be very sensitive to the window length (6 cycles in this case) as well as the window type (flat top in this case).

Figures 11 to 13 show the results for a dynamic compliance test using phase modulation. In this test, the bandwidth of the PMU is investigated. To this end the phase of the fundament is modulated with a frequency ranging from 0.1 to $5 \mathrm{~Hz}$ whose amplitude is +/- 0.1 radian. The measurements show that the modulation has a limited effect on the TVE, but impacts the frequency and ROCOF in a much stronger fashion. The device is nevertheless compliant.

Figure 14 shows the step response of the PMU to a phase jump of $10^{\circ}$. Due to the $20 \mathrm{~ms}$ time interval between two PMU measurements (frame rate of $50 \mathrm{fps}$ ), very few samples are available during the transient. To circumvent this limitation, interleave sampling technique is used in which the test is repeated several times, but with the step being slightly diplaced with respect to the reporting time. The result is an higher sampling rate.

\section{PMUs in distribution networks}

While PMUs are accepted as an instrument in transmission systems, they are generally not perceived as suitable for distribution networks. The small size of distribution networks result in small phase displacements. Additionally, the level of power quality disturbances is much higher due to the proximity of the loads. Nevertheless, PMU functionalities could contribute to the control of networks with a high penetration of distributed generation.

The requirements, in term of TVE, for the usage of PMUs in distribution are not straight forward to estimate. The laboratory of Distributed Electrical Systems Laboratory at the EPFL (Ecole Polytechnique Fédérale de Lausanne) investigated these requirements for state estimation applications and power flow monitoring. To be exploitable, the accuracy of the power flow must be at least $10 \%$ which require PMUs with a TVE error in the order of $0.3 \%$ [8]. However, other applications such as active voltage control or protection are likely to require even smaller errors. This places even more stringent requirements on the test equipment. 
Table 1 shows the error allowed on magnitude, phase or timing (single source of error) for a given TVE. The table shows a 5 fold improvement at each line. This is also the minimum ratio of the accuracy of a calibrator and the device under test. The first line corresponds to the requirements of $1 \%$ TVE set by C37.118.1. However, TVE around $0.05 \%$ (or better) are often being articulated for applications in distribution networks.

Table 1. Magnitude, phase and time accuracy

\begin{tabular}{lcccc}
$50 \mathbf{H z}$ & $\begin{array}{c}\text { TVE } \\
\%\end{array}$ & $\begin{array}{c}\text { Mag. } \\
\text { ppm }\end{array}$ & $\begin{array}{c}\text { Phase } \\
\text { deg }\end{array}$ & $\begin{array}{c}\text { Time } \\
\mu \mathrm{s}\end{array}$ \\
\hline \hline & & & & \\
C37.118.1 & 1 & 10,000 & 0.57 & 32 \\
& 0.2 & 2,000 & 0.11 & 6.4 \\
& 0.04 & 400 & 0.022 & 1.3 \\
& 0.008 & 80 & 0.0046 & 0.25 \\
& 0.0016 & 16 & 0.00092 & 0.050
\end{tabular}

To address the accuracy upgrade, a strategy has been devised along the degree of difficulty. The first is to minimise the timing error. As shown in Figure 4, GPS is affected by time fluctuations of around $+/-20 \mathrm{~ns}$. But, when two receivers (PMU and its calibrator) are used, the two time fluctuations are not coherent even if the antennas of the two receivers point toward the same sky. Figure 15 shows this time difference between the two receivers. The deviation can be up to $70 \mathrm{~ns}$. To circumvent this difficulty, the calibrator is now connected directly to UTC-CH. This easy modification has permitted to reduce the time jitter from 20 to about $0.2 \mathrm{~ns}$.

The accuracy of the magnitude and phase are being upgraded by means of an active calibration and the selection of ADCs and DACs with low jitter. The active calibration will be implemented for the voltage path by using a high accuracy HP 3458 multi-meter whose sampling is coherent with the calibrator's clock circuits.

The PMUs to be used in distribution networks will require new test scenarios linked primarily with power quality. These tests will require special waveforms with

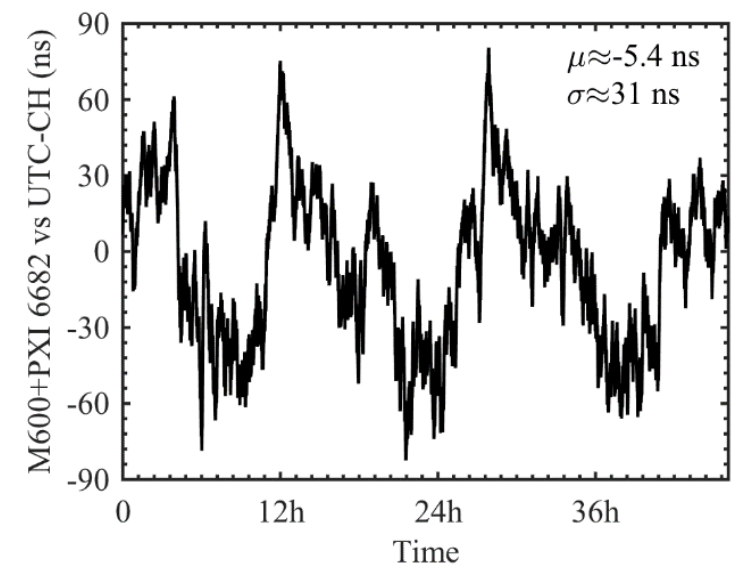

Figure 15. Combined uncertainty of two arbitrary GPS receiveirs: Manberg LANTIME M600 and PXI-6682. disturbance levels equal or exceeding those of the standard EN 50160 [9]. To this end a real time waveform generator is implemented which is capable of following a scripted description of a PQ level or event.

\section{Conclusions}

This paper presented the design of a PMU calibrator that has been developed at METAS as well as some measurement results obtained with a commercial PMU designed around the standards IEEE C37.118.1. The ongoing improvement that is presently taking place aims at making this calibrator suitable for future PMUs likely to be used in distribution networks. The use of an atomic clock, active magnitude calibration and better converters will reduce the calibrator's TVE in the vicinity of $0.01 \%$.

In addition of the calibrator's accuracy, a suite of new tests will also be designed to ensure that PMUs used in distribution networks continue to operate properly in the presence of power quality disturbances.

Combined, this advanced calibrator will provide a metrological platform for distribution networks PMUs.

\section{Acknowledgment}

This work received funding through the European Metrology Research Programme (EMRP), Project ENG04 'Metrology for Smart Electrical Grids'. The EMRP is jointly funded by the EMRP participating countries within EURAMET and the European Union.

\section{References}

1. M. Kezunovic et al., Application of time-synchronized measurements in power system transmission networks, Springer (2014)

2. J. Depablos et al., IEEE Power Engineering Society General Meeting, 1, 948, (2004)

3. W. Dickerson, Establishing traceability of synchronised phasor measurements at the national laboratory level, Securing critical infrastructure conference, (2004)

4. IEEE Standard for Synchrophasor. Measurements for Power Systems, IEEE Std C37.118.1, (2011)

5. IEEE Standard for Synchrophasor Measurements for Power Systems, Amendment 1: Modification of Selected Performance Requirements, IEEE Std C37.118.1a, (2014)

6. G.N Stenbakken, T. Neslon, IEEE Power Engineering Society General Meeting, 3, paper 5, (2006)

7. G. Rietveld et al., IEEE Transactions on Instrumentation and Measurement, 6, 1355, (2015)

8. D. Colangelo et al., Architecture and characterization of a calibrator for PMUs operating in power distribution systems, PowerTech conference, (2015)

9. Voltage characteristics of electricity supplied by public distribution system, Standard EN 50160, (2010) 